\title{
Brief psychotic disorder due to ephedrine
}

\author{
Isik Batuhan Cakmak ${ }^{1 \oplus}$, Ayse Gulden Gurses ${ }^{1 \oplus}$, Hasan Kaya $^{1 \oplus}$, Erol Goka ${ }^{1 \odot}$ \\ 'University of Health Sciences, Ankara City Hospital, Department of Psychiatry, Ankara - Turkey
}

\section{Dear Editor,}

Ephedrine is an alkaloid derived from an evergreen shrub known as Ephedra (or Ma Huang), traditionally known in China for over 5000 years and used in alternative medicine $(1,2)$. The Ephedra Sinica strain used for therapeutic purposes is called Ma Huang, which means "yellow hemp" in Chinese. Tea obtained by boiling the plant contains about $1.5-9.0 \mathrm{~g}$ of ephedrine (2). The traditional properties of ephedrine used in alternative medicine were described by the Chinese Emperor Shen Nung (3) and were often used for cognitive-stimulation, sexual performance enhancement, and weight loss. It has also been reported to be used in cases such as allergic rhinitis, asthma, influenza, fever, hypotension, spinal anesthesia, flu, nasal congestion, narcotic antidote, Adams-Stokes syndrome and dysmenorrhea $(1,2,4,5)$.

The main pharmacological effect of ephedrine is sympathomimetic. Its molecular structure is the $\beta$ hydroxy analogue of methamphetamine and therefore has an agonist effect on both $\alpha$ and $\beta$ adrenergic receptors. Although its central stimulant effect is much less effective than methamphetamine, it is more effective on $\alpha$ and $\beta$ adrenergic receptors $(1,3)$. It has structurally direct or indirect effective sympathomimetic properties and is a substrate of the noradrenaline transporter at synapses such as other sympathomimetic agents like amphetamine, methamphetamine and tyramine (2). Through this mechanism of action, ephedrine increases heart rate and cardiac output; increases peripheral resistance, thus blood pressure, stimulates bronchodilatation due to its agonist effect on $\beta$-adrenergic receptors and has therefore been used in relieving asthmatic symptoms $(1,2)$. It causes stimulation in the cortical and subcortical regions and shows its effect in narcolepsy and depressive states in this way. Signs and symptoms such as irritability, anxiety, fear, tension, agitation, excitation, restlessness, weakness, increased speech, impulsivity can be detected as a result of their effects on the central nervous system (2).

Psychiatric symptoms due to ephedrine use include psychotic symptoms and mood symptoms. Schizophrenia-like psychosis caused by stimulants is thought to be due to neuronal degeneration in the dopaminergic system. The hypodopaminergic condition following a previous hyperdopaminergic condition that causes symptoms similar to positive schizophrenic symptoms is held responsible (6).

A 33-year-old single, male patient living with his mother and engaged in photography was admitted to our clinic with a pre-diagnosis of psychotic disorder. Having no psychiatric diagnosis and treatment before, the patient had been confusing the words in his mind, thinking that his thoughts were manipulated by an outer force for about 6 months. According to his relatives, the patient was always nervous and had meaningless speech, some of which were while on his own.

The mother of the patient was followed up for obsessive-compulsive disorder and was on fluoxetine $60 \mathrm{mg} /$ day, aripiprazole $10 \mathrm{mg} /$ day, and her father had alcohol use disorder but had not been treated.

The patient had first started using ephedrine at the age of 15 to improve his sports performance while

How to cite this article: Cakmak IB, Gurses AG, Kaya H, Goka E. Brief psychotic disorder due to ephedrine. Dusunen Adam The Journal of Psychiatry and Neurological Sciences 2020;33:441-443.

Correspondence: Isik Batuhan Cakmak, University of Health Sciences, Ankara City Hospital, Psychiatry Department, No: 1, Cankaya, Ankara - Turkey E-mail: batuhancakmak@hotmail.com 
playing football professionally. He had been using ephedrine tablets (150-200 mg/day) for 15 years and ephedrine ampoules for the last 6 years. The last time he used ephedrine was the day before his admission. He did not share the method and dose of ephedrine with the treatment team.

The patient, who visited out of the city due to his profession in the summer, cut the sofas in the place where he lived 4 months ago and thought that he was being chased by demons and broadcasted. He thought that one of his friends was manipulating the words in his mind, changing the meaning of the words, and some implying negative thoughts about him. He had been suffering from insomnia for about 4 months and started to spend most of his time at home. He began to think that the tenants were cooperating and making plans with the government officials and his mother. The patient also described the auditory and tactile hallucinations and explained these hallucinations with accompanying delusions.

No pathology was found in the patient's complete blood count, routine biochemistry and urine tests. Magnetic resonance imaging and electroencephalogram were normal. The patient had delusions and hallucinations in the clinic and risperidone treatment was initiated with a prediagnosis psychotic disorder.

During the first days of his hospitalization, the patient had a severe headache and asked for intense painkillers. He was consulted to the neurology department and no pathology was reported. The psychotic symptoms of the patient rapidly regressed and $4 \mathrm{mg}$ risperidone was continued throughout his hospitalization. The patient was followed up in our clinic for 12 days. In the mental state examination at discharge, the patient was conscious, oriented towards himself and his environment, and was able to communicate with the interviewer. His self-care and outward appearance were consistent with his social and cultural level. His emotion was dull and his mood was euthymic. His thought content included themes from daily life, and paranoid delusions were limited. Schneiderian symptoms were not observed. Psychomotor activity was within normal limits. The patient was discharged on the 12th day of hospitalization with $4 \mathrm{mg}$ risperidone. There were no psychotic findings in the follow-up after discharge. Risperidone dose was reduced and discontinued during follow-up. The patient authorized the corresponding practitioner and other co-authors to write a case report about his treatment.
Ephedrine-induced psychosis was first defined in 1968 by Herridge and O'Brook (7) and many case reports and series were published in the following years (4,5,7-9). In these publications, paranoid symptoms were prominent in the manifestation of ephedrine-induced psychotic disorder, and torment and grandiose delusions were frequently accompanied in addition to the increased speed and amount of speech, increased anger, irritability, impulsive behaviors, and auditory hallucinations. When the cases are examined, it can be observed that patients can be grouped into four categories. Some of the patients show psychiatric symptoms due to chronic treatment for another disease, some non-prescription abuse without a psychiatric disorder, some seek only the central stimulating effect of ephedrine and some have psychiatric symptoms due to the interactions with other stimulants. Our case is in the second group because of the abuse of prescription drugs that increase sports performance and bodybuilding.

In our patient, the onset of psychotic symptoms after long-term ephedrine abuse, discontinuation of ephedrine use and regression of symptoms with antipsychotic treatment, and the absence of a previously known psychiatric diagnosis suggested that psychotic features were related to ephedrine use.

In recent years, ephedrine-containing products have been frequently marketed, and especially targeted people who want to lose weight or are interested in bodybuilding $(2,9,10)$. The amount of ephedrine found in energy drinks is typically stated as $20 \mathrm{mg}$, but varies from product to product. Studies in animals show that high doses of ephedrine cause symptoms of anxiety and restlessness due to sympathetic stimulation, and muscle tremors and seizures may develop as doses increase. In humans, a dose of $50 \mathrm{mg} /$ $\mathrm{kg}$ was determined to be potentially lethal, but symptoms of intoxication were observed at doses 3-4 times the therapeutic dose of $150 \mathrm{mg} /$ day. Ephedrine was described as a non-abusive substance in the past, but its access was still limited. Especially, in 1994, Food and Drug Administration and Centers for Disease Control and Prevention reviewed more than 100 deaths and nearly 500 side effect reports over 2 years, and took measures to inform healthcare workers, raise awareness, and limit its access $(2,4,9)$.

In order not to overlook ephedrine-related psychiatric disorders a detailed history should be taken from the patient. The nutritional duration, amount, frequency and purpose of substances like $\mathrm{Ma}$ Huang should be questioned in detail. Thus, it may be 
possible to detect self-medication or a treatable psychiatric condition that is not understood with the help of other symptoms. The patient may be using products for lack of energy, fatigue, hypersomnia or depression. Hence, a primary medical or psychiatric disorder that has not yet been diagnosed or missed can be elucidated (9).

In ephedrine-induced psychotic disorders and mood disorders, supportive treatment should be a priority. Psychotic symptoms and agitation may require the use of antipsychotics, benzodiazepines, or both at the lowest possible doses, taking side effects into account. The patient may need to be hospitalized in the case of mania, severe psychotic symptoms, possible suicides or harmless behaviour towards the environment. Close cardiac monitoring and physical examination, especially for the possible arrhythmias, are important in these patients. It should be kept in mind that symptoms may last for days, weeks or even months after ephedrine is discontinued $(9,11)$.

Informed Consent: The patient has authorized the corresponding practitioner and other co-authors to write a case report about his treatment.

Conflict of Interest: We, the authors of this manuscript, declare that we have no conflicts of interest.

Financial Disclosure: This article did not receive any specific grant from funding agencies in the public, commercial, or not-for-profit sectors.

\section{REFERENCES}

1. Solanki P, Yadav P, Kantharia N. Ephedrine: direct, indirect or mixed acting sympathomimetic? Int J Basic Clin Pharmacol 2014; 3:431-436. [CrossRef]

2. Vaidya VS, Mehendale HM. Ephedra: In Wexler P (editor). Encyclopedia of toxicology. Third ed. Oxford: Academic Press, 2014; 426-430. [CrossRef]

3. Uzbay IT. Substance abuse - all aspects of addiction and addictive substances. Istanbul Tip Kitabevi, 2015. (Turkish)

4. Walton R, Manos GH. Psychosis related to ephedra-containing herbal supplement use. South Med J 2003; 96:718-720. [CrossRef]

5. Roxanas MG, Spalding J. Ephedrine abuse psychosis. Med J Aust 1977; 2:639-640. [CrossRef]

6. Lieberman JA, Kinon BJ, Loebel AD. Dopaminergic mechanisms in idiopathic and drug-induced psychoses. Schizophr Bull 1990; 16:97-110. [CrossRef]

7. Herridge CF, O’Brook MF. Ephedrine psychosis. Br Med J 1968; 2:160. [CrossRef]

8. Peterson E, Stoebner A, Weatherill J, Kutscher E. Case of acute psychosis from herbal supplements. S D Med 2008; 61:173-177.

9. Jacobs KM, Hirsch KA. Psychiatric complications of Ma-huang. Psychosomatics 2000; 41:58-62. [CrossRef]

10. Miller SC. Psychiatric effects of ephedra: addiction. Am J Psychiatry 2005; 162:2198. [CrossRef]

11. Mullen JM, Richards JR, Crawford AT. Amphetamine related psychiatric disorders. https://www.ncbi.nlm.nih.gov/books/ NBK482368/. Accessed September 1, 2020. 